This item was submitted to Loughborough's Research Repository by the author.

Items in Figshare are protected by copyright, with all rights reserved, unless otherwise indicated.

\title{
A non-contact opto-electronic sensing device for precision linear and angular positioning of robotic manipulators
}

PLEASE CITE THE PUBLISHED VERSION

PUBLISHER

Professional Engineering Publishing / @ IMECHE

VERSION

VoR (Version of Record)

LICENCE

CC BY-NC-ND 4.0

\section{REPOSITORY RECORD}

Shams, I., Homer Rahnejat, and D. Greening. 2019. "A Non-contact Opto-electronic Sensing Device for Precision Linear and Angular Positioning of Robotic Manipulators". figshare. https://hdl.handle.net/2134/4820. 
This item was submitted to Loughborough's Institutional Repository (https://dspace.lboro.ac.uk/) by the author and is made available under the following Creative Commons Licence conditions.

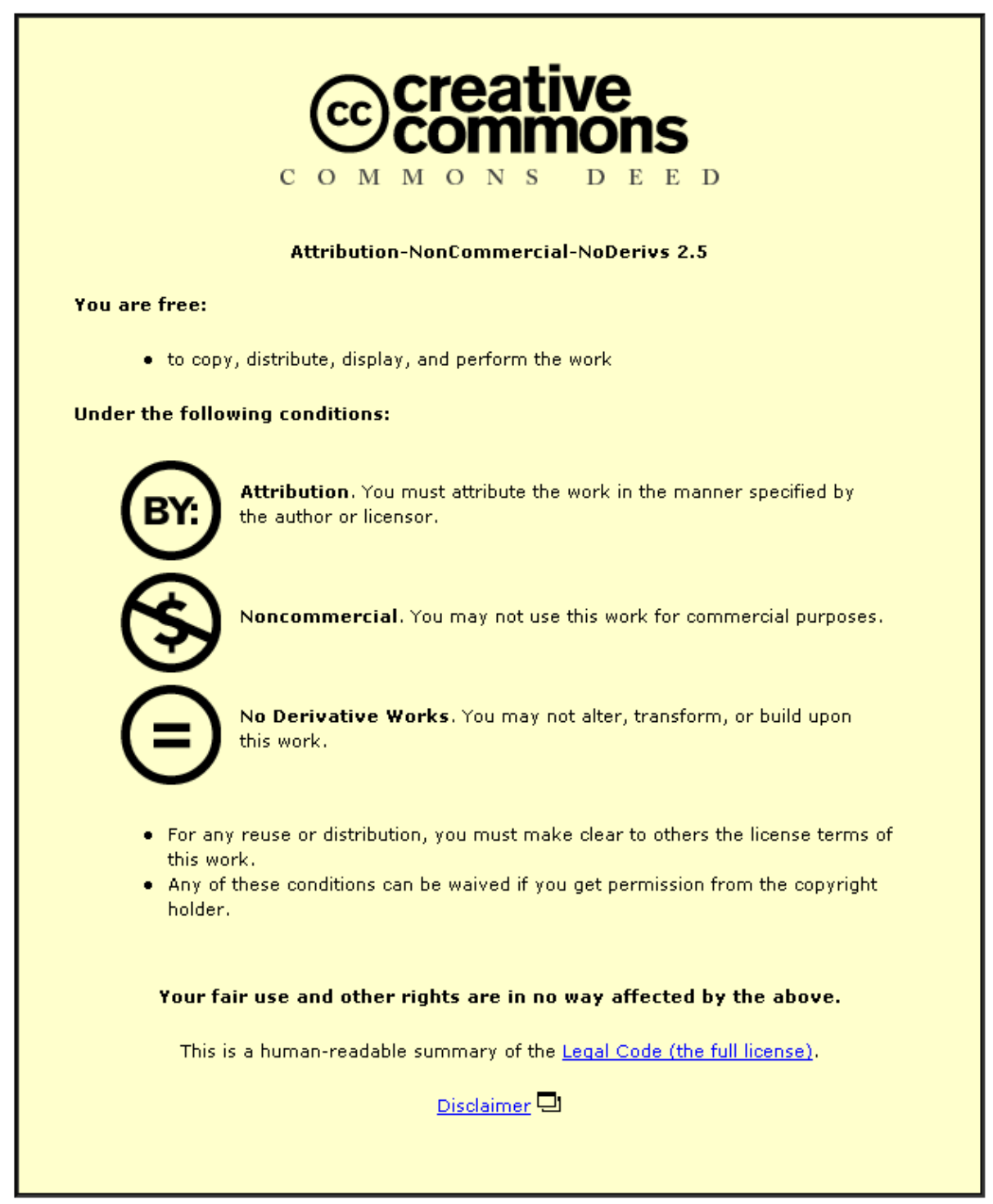

For the full text of this licence, please go to: http://creativecommons.org/licenses/by-nc-nd/2.5/ 


\title{
A non-contact opto-electronic sensing device for precision linear and angular positioning of robotic manipulators
}

\author{
I Shams, BSc, MCybS \\ Department of Manufacturing and Engineering Systems, Brunel, the University of West London, Uxbridge, Middlesex \\ H Rahnejat, MSc, PhD, DIC, CEng, MIMechE, FCybS \\ School of Mechanical, Aeronautical and Production Engineering, Kingston Polytechnic, Kingston, Surrey \\ D Greening, BSc, MSc \\ $\mathrm{V}$ and S Scientific (London) Limited, Brosnan House, Darkes Lane, Potters Bar, Hertfordshire
}

A non-contacting optical sensor is devised to monitor the position and orientation of a robot end-effector or grasped object within fine limits of accuracy. This enables fine alignment of tools or parts with respect to assembly fixtures, thus rendering precision assembly tasks to be performed in a dexterous manner. The sensor is capable of detecting linear displacements of the order of $5 \mu \mathrm{m}$ or better and angular misalignments down to 5 seconds of arc. The principle of differential detection is employed which reduces the undesired effects of noise and fluctuations in the source intensity.

\section{NOTATION}

$\begin{array}{ll}a & \text { slit width } \\ b & \text { slit length } \\ I & \begin{array}{l}\text { irradiance intensity } \\ \text { image length } \\ n\end{array} \\ N, M & \begin{array}{l}\text { coordinate of a point within the strip image } \\ \text { with respect to its centre }\end{array} \\ N & \text { numerical aperture } \\ p & \text { total beam power } \\ \text { transmitted power } \\ t & \begin{array}{l}t=\sqrt{2} M / w \\ \text { beam radius }\end{array} \\ w & \text { slit coordinate system (displacements) } \\ X, y, z & \text { robot coordinate system } \\ X, Y, Z & \text { yaw axis } \\ \beta & \text { pitch axis } \\ \gamma & \text { roll axis } \\ \theta & \text { skew angle between the emitting strip image } \\ \lambda & \text { and the slit } \\ \lambda & \text { laser diode wavelength }\end{array}$

\section{Subscripts}

0 refers to the centre of the image or the centre of the beam

M refers to any position in the $M$ direction along the width of the strip image

\section{INTRODUCTION}

Not until recently have opto-electronic sensing devices been used in sophisticated robotic applications. The chief reason for this has been the rather primitive state of industrial robot applications such as paint spraying, palletizing and depalletizing, spot welding and machine loading, all of which require little sensory interaction

The MS was received on 14 December 1987 and was accepted for publication on 19 August 1988. between robots and their work environment $(1,2)$. Not suprisingly, first generation robots (with little or no external sensing) have been predominantly employed. Therefore, in machine loading/unloading operations and simple repetitive robot assembly tasks the environment is strictly structured to guard against accidental disorientation of parts. The assembly processes are either permanent, using adhesives, rivets, etc. or non-permanent, employing guiding elements to effect the assembly of mating parts by dead reckoning.

Such approaches, however, are unlikely to extend the sphere of application of robotics to the flexible product assembly area where their greatest potential must surely lie. Two major requirements exist for successful implementation of such systems. Firstly, the ability of robots should be extended in such a way as to interact with variable unstructured environments that are prevalent in manufacturing systems (3). Robots should, therefore, possess adequate perceptual powers to sort and identify parts that are not strictly regimented in an objectorientated environment such as out of a bin or from a conveyor. This task is usually allocated to vision and tactile sensing systems that are developed and continually enhanced $(4,5)$. Secondly, it is important to enhance the positional repeatability of robots in order to meet the tight tolerances that are often required to effect precision assembly tasks.

Opto-electronic devices have been traditionally used in robotic applications for such tasks as detection of a target (6), range finding, tracking (7) or obstacle avoidance. In these applications their precise measuring accuracy is not fully exploited. Yet some recent works have shown the potential of these devices both for recognition of objects and discrimination of object features, for instance when they are incorporated within a tactile sensing measuring structure (8). They have also been employed with touch sensing configurations for detection of slip during the process of object manipulation (9). 


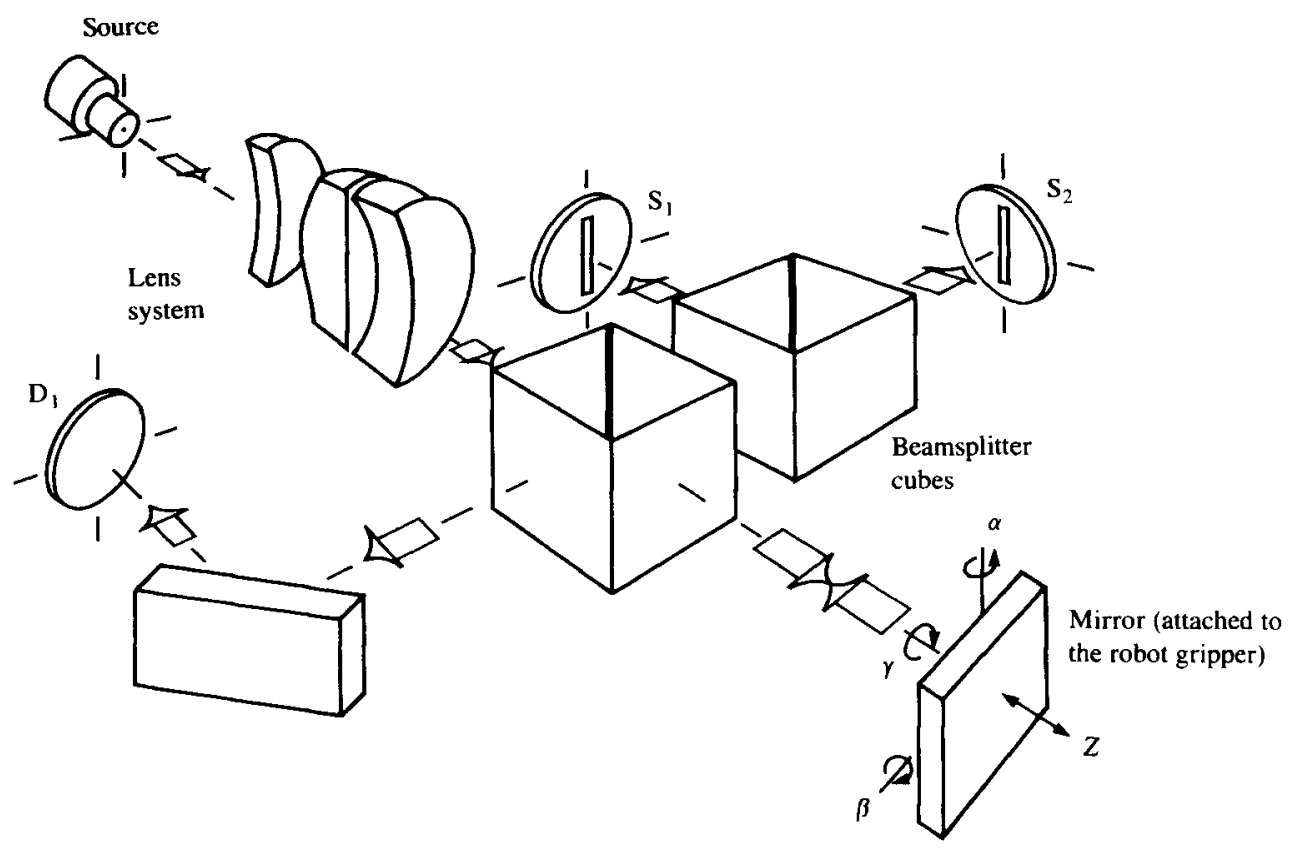

Fig. 1 Schematic representation of the opto-electronic sensing device; the motions detected are in the $Z$ and $\alpha$ directions

Opto-electronic devices can also be used for detection of position and orientation of parts prior to placement or insertion work. In this role they can be best used with robots to improve their positioning and alignment accuracy to meet the tight tolerances of precision product assembly work. This paper outlines a laserassisted device that can be used to provide feedback information to a robotic micro-manipulator attached to the wrist of a robot to correct its position and pose in order to meet the task of assembly of closely toleranced products.

\section{PRINCIPLES OF MEASUREMENT}

Figure 1 depicts a schematic presentation of the principles of measurement employed by the opto-electronic device. The source used is a laser diode with a $850 \mathrm{~nm}$ pulsed window output. This in fact falls within the peak response region of the silicon photodiode since the wavelength determines the depth of penetration of photons into the silicon structure (10).

The laser diode is used because of its small size and modest power requirements compared with other lasers. However, the output radiation from a laser diode is highly divergent which is a result of its miniscule emitting source dimensions (of the order of the wavelength of the radiation, $750-1700 \mathrm{~nm})(11)$.

In order to collect as much of the radiation from the laser diode as possible and to realize its full potential in terms of producing a sharply focused image, a set of associated optics are required. These optics must be of high quality.

The laser beam is directed towards the target surface through a beamsplitter. A proportion of the beam power is diverted to detector $\left(D_{1}\right)$ which continuously monitors the output power fluctuations. The onward beam is reflected back from the target surface and is redirected by the same beamsplitter to a differential detection unit. This unit consists of a further beamsplitter and a pair of detecting elements.

Each detecting element is preceded by a slit which defines the spatial location of power reaching the silicon surface of the detector. The beamsplitter, nominally $50 / 50$, divides the paths forming two images at slits $S_{1}$ and $S_{2}$ (see Fig. 2a). The slits are at slightly different focal distances, the resulting situation is represented by Fig. $2 b$ with slit $S_{1}$ shown as a virtual image. The detectors' outputs are 'equal' only when the image plane is

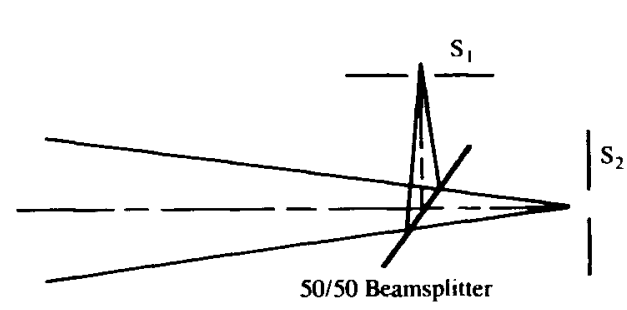

Fig. 2a Slits at different focal distances

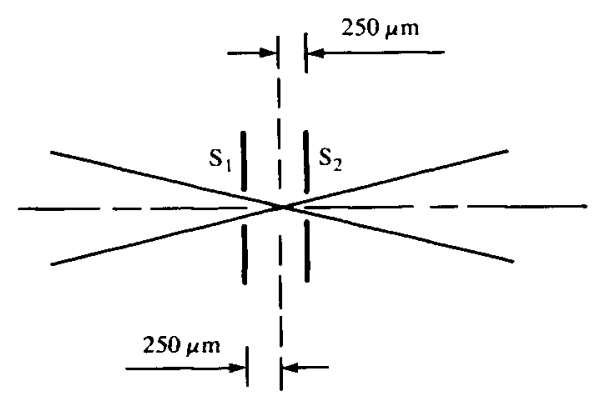

Fig. 2b Slits arranged with focus at null point

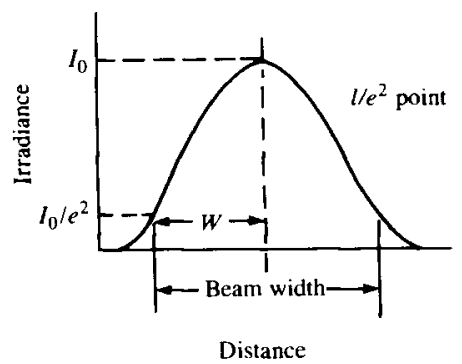

Fig. 2c Gaussian beam profile 
mid-way between them. This is referred to as the calibrated null point which can be detected with extreme accuracy (12). This detection procedure is independent of the laser power (within reason), allowing the most inexpensive combination of laser/power supply. It also partially decouples the angular and linear measurements, thereby reducing the burden of signal processing.

\section{DETERMINATION OF SLIT WIDTH}

The irradiance distribution of a laser diode beam can be assumed to propagate as a Gaussian beam in the $\mathrm{TEM}_{00}$ mode $(13,14)$. The intensity profile of the beam is therefore given by (see Fig. 2c) (14)

$$
I_{\mathrm{M}}=I_{0} \exp \frac{-2 M^{2}}{w^{2}}
$$

The beam intensity profile given is across a long narrow image, where $I_{0}=(2 / \pi)^{1 / 2} \cdot p / w$ and is the irradiance intensity at the centre of the beam and $w$ is the beam radius

$$
w=w_{0}\left\{1+\left(\frac{\lambda z}{\pi w_{0}}\right)^{2}\right\}^{1 / 2}
$$

where: $w_{0}=\lambda / \pi$ (NA) and $z$ is the axial displacement.

The transmitted power through the slit is determined by

$$
s=\int_{-\infty}^{+\infty} I_{M} \mathrm{~d} M
$$

However, the power transmitted through a slit of finite width $a$ is

$$
s=\int_{-a / 2}^{a / 2} I_{M} \mathrm{~d} M=2 \int_{0}^{a / 2} I_{M} \mathrm{~d} M
$$

Substituting for $I_{M}$ from equation (1) yields the following:

$$
s=2\left(\frac{2}{\pi}\right)^{1 / 2} p / w \int_{0}^{a / 2} \exp \frac{-2 M^{2}}{w^{2}} \mathrm{~d} M
$$

The integral in equation (5) is solved by the substitution

$$
t=\sqrt{2} M / w
$$

and using the error function

$$
\operatorname{erf}(t)=\frac{2}{\sqrt{\pi}} \int_{0}^{t} \exp \left(-t^{2}\right) \mathrm{d} t
$$

which yields

$$
s=p \cdot \operatorname{erf} \frac{a}{\sqrt{2} w}
$$

Substituting for $w$ from equation (2) and solving equation (7) for the ratio $s / p$ in terms of $z$ yields the results illustrated in Fig. 3. Three characteristic curves for different widths of slit are shown in this figure. The lower curve indicates that only 80 per cent of power could be transmitted at the position given by $z=0$ which is suboptimal. It also indicates a small variation in the ratio of $s / p$ for the values of $z$ exceeding $175 \mu \mathrm{m}$ which provides a small operating range for positional errors usually associated with precision robotic work such as close fit product assembly. On the other hand the upper curve shows small changes in the value of the ratio $s / p$

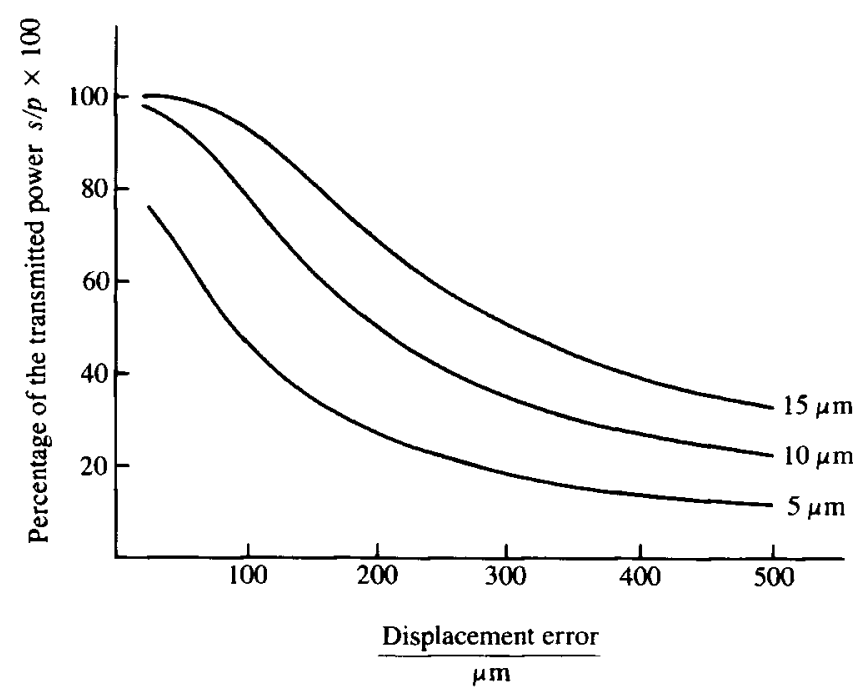

Fig. 3 Characteristic curves giving measurement sensitivity with linear errors for various slit widths

in the region of small values of $z$ (that is $<100 \mu \mathrm{m}$ ). The repeatability of robots suitable for precision assembly tends to be in the region of $25-250 \mu \mathrm{m}$, thus making the middle graph the most suitable to be used for the purpose of detection of errors with the sensing system (1). Figure 4 shows the typical error history of a precision robotic manipulator when subjected to pseudorandom manoeuvres between two pre-specified locations.

The history shown in this figure relates to the translational errors incurred by the manipulator in reaching a commanded position. In addition to these there are misalignment or out of orientation errors which are associated with the pose of the gripper or the tool held with respect to the commanded position to be achieved or the task to be performed. These latter errors are usually more critical in determining the suitability of the robot in performing precision assembly work such
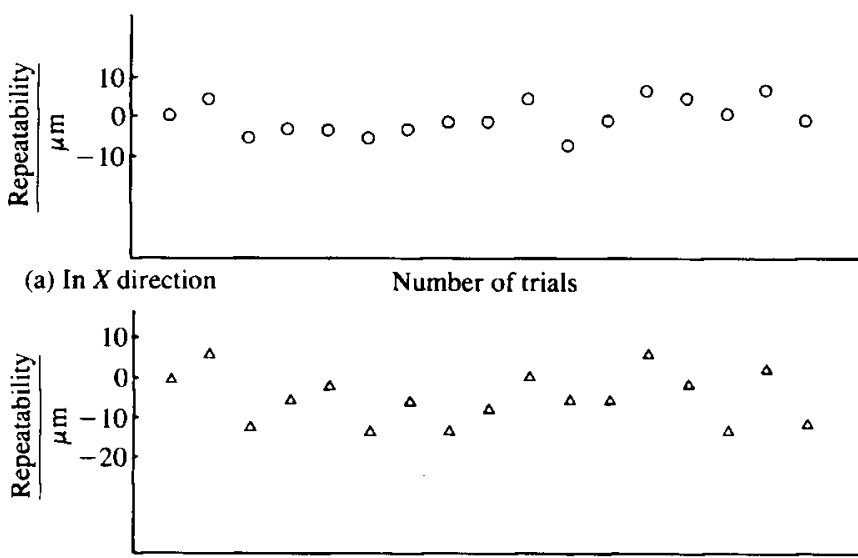

(b) In $Y$ direction Number of trials

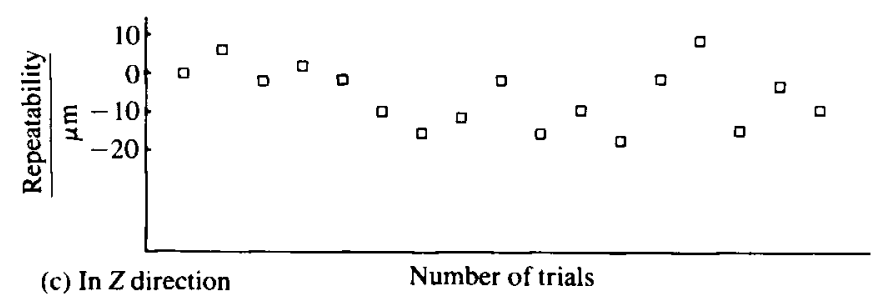

Fig. 4 Error history of the tool centre point of a precision $T^{5}$ robotic manipulator

Proc Instn Mech Engrs Vol 202 No B4 
as shaft and bearings and turbine blades onto the rotor disc. Tests carried out show that in the latter case the fitting translational tolerance (free of angular misalignment) is approximately $75 \mu \mathrm{m}$. However, in the presence of orientational errors the maximum allowable translational error is further reduced to $25 \mu \mathrm{m}$. Therefore, to make certain that the choice of slit width meets the demands imposed by the orientation errors the characteristic reponses of the $10 \mu \mathrm{m}$ and $15 \mu \mathrm{m}$ slits should be further investigated.

\section{ANGULAR AND LINEAR SENSITIVITY OF THE SENSING DEVICE}

Referring to Fig. 1, the orientation of the gripper is given by the roll-pitch-yaw rotations of the robot wrist. Pitch, denoted by $\beta$, causes the source image to be shifted up and down in the direction of the slit length. Since the slit is of sufficient length the displaced image is easily accommodated. Roll, denoted by $\gamma$, causes no change in the position of the reflected image and is therefore undetected. On the other hand rotation about the yaw axis, denoted by $\alpha$, shifts the reflected beam in the lateral direction to the slits longitudinal axis. The disposition of the focal point is directly affected by the normal displacement of the target surface, given by $Z$. The effect of these two sources of error are discussed in this section.

The positional vector of any point on the strip image with coordinates $(N, M)$ relative to the slit coordinate system $x, y$ is represented in the following form (see Fig. 5):

$$
\left[\begin{array}{l}
N \\
M
\end{array}\right]=\left[\begin{array}{rr}
\cos \theta & \sin \theta \\
-\sin \theta & \cos \theta
\end{array}\right]\left[\begin{array}{l}
\left(x-x_{0}\right) \\
\left(y-y_{0}\right)
\end{array}\right]
$$

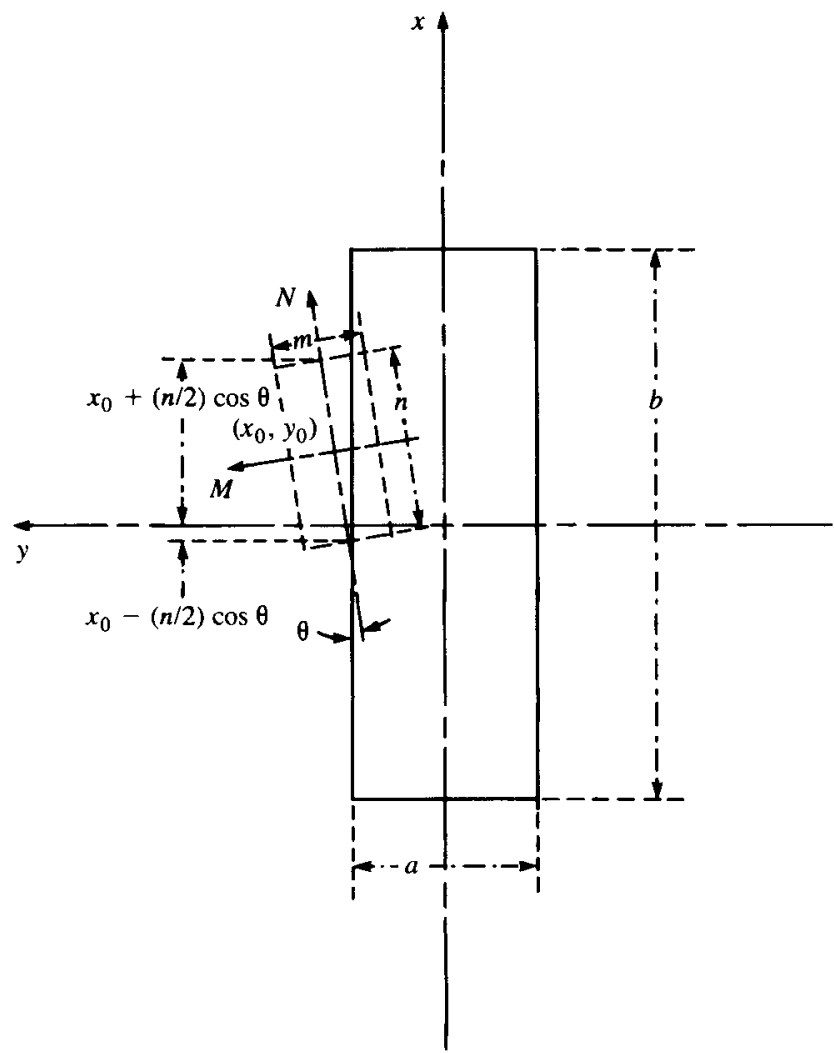

Fig. 5 Skewed strip image representation with respect to the slit where $\theta$ is the skew angle and $x_{0}, y_{0}$ denote the centre of the strip image. This skew is caused by misalignment between the laser source and the slit.

Irradiance intensity at any point on the image $(N, M)$ is given by equation (1). In order to find the total transmitted power $s$ of the two-dimensional image the following integral is evaluated:

$$
s=\int_{x_{0}-1 / 2 n \cos \theta}^{x_{0}+1 / 2 n \cos \theta} \int_{-a / 2}^{a / 2} I(x, y) \mathrm{d} x \mathrm{~d} y
$$

Therefore, replacing for irradiance $I$ from equation (1):

$$
\begin{array}{r}
s=\int_{x_{0}-1 / 2 n \cos \theta}^{x_{0}+1 / 2 n \cos \theta} \int_{-a / 2}^{a / 2} I_{0} \exp \left\{-2 / w^{2}\left[-\left(x-x_{0}\right)\right.\right. \\
\left.\left.\times \sin \theta+\left(y-y_{0}\right) \cos \theta\right]^{2}\right\} \mathrm{d} x \mathrm{~d} y
\end{array}
$$

Integrating with respect to $x$ and $y$, replacing for $I_{0}$ in terms of the laser diode's output power and the beam radius, for values of $\theta$ smaller than one degree the following solution results:

$$
s=(2 / \pi)^{1 / 2} n p / w \int_{-a / 2}^{a / 2} \exp \left\{-2\left(y-y_{0}\right)^{2} / w^{2}\right\} d y
$$

Using Simpson's rule the above integral is solved to give the percentage power transmitted against the lateral position of the image centre, $y_{0}$. Figure 6 shows the theoretical characteristic curves for a slit width of $10 \mu \mathrm{m}$ at different values of translational error. The characteristic curves indicate large variations in the transmitted power for small changes in the misalignment angle of the reflective surface given by the yaw angle, $\alpha$. The percentage power transmitted falls with

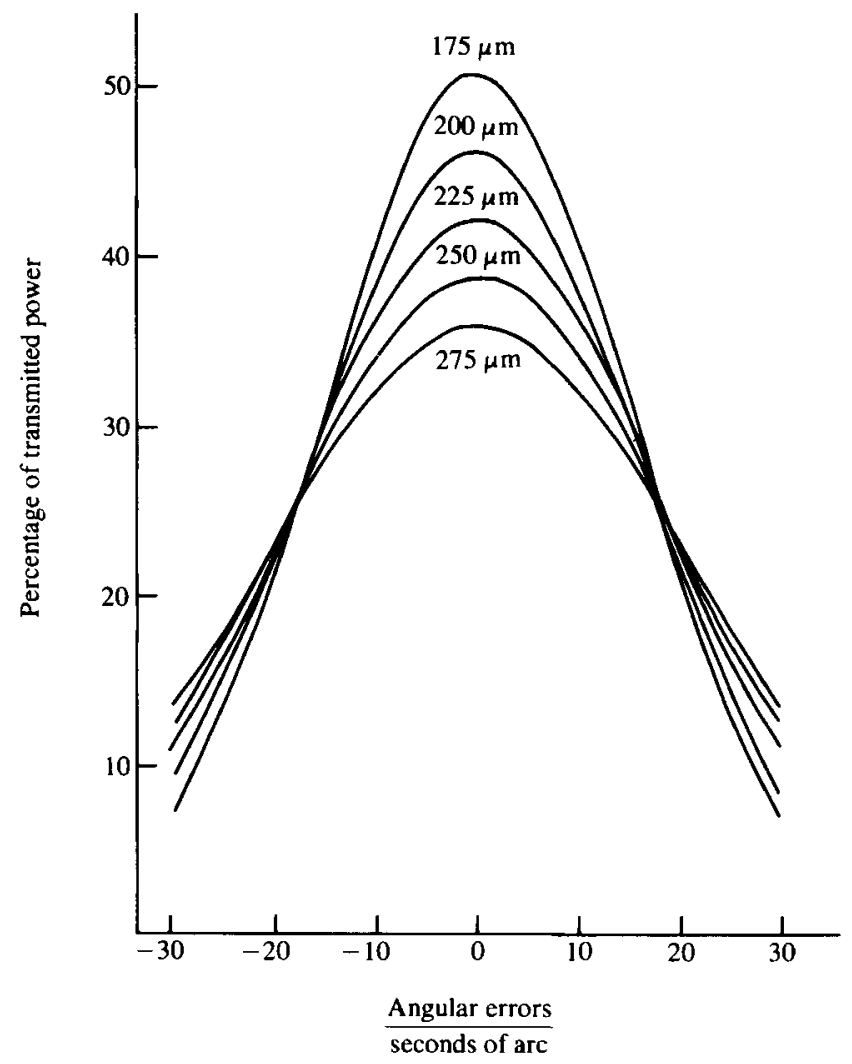

Fig. 6 Characteristic curves giving measurement sensitivity with angular errors for various linear errors $(10 \mu \mathrm{m}$ slit) 


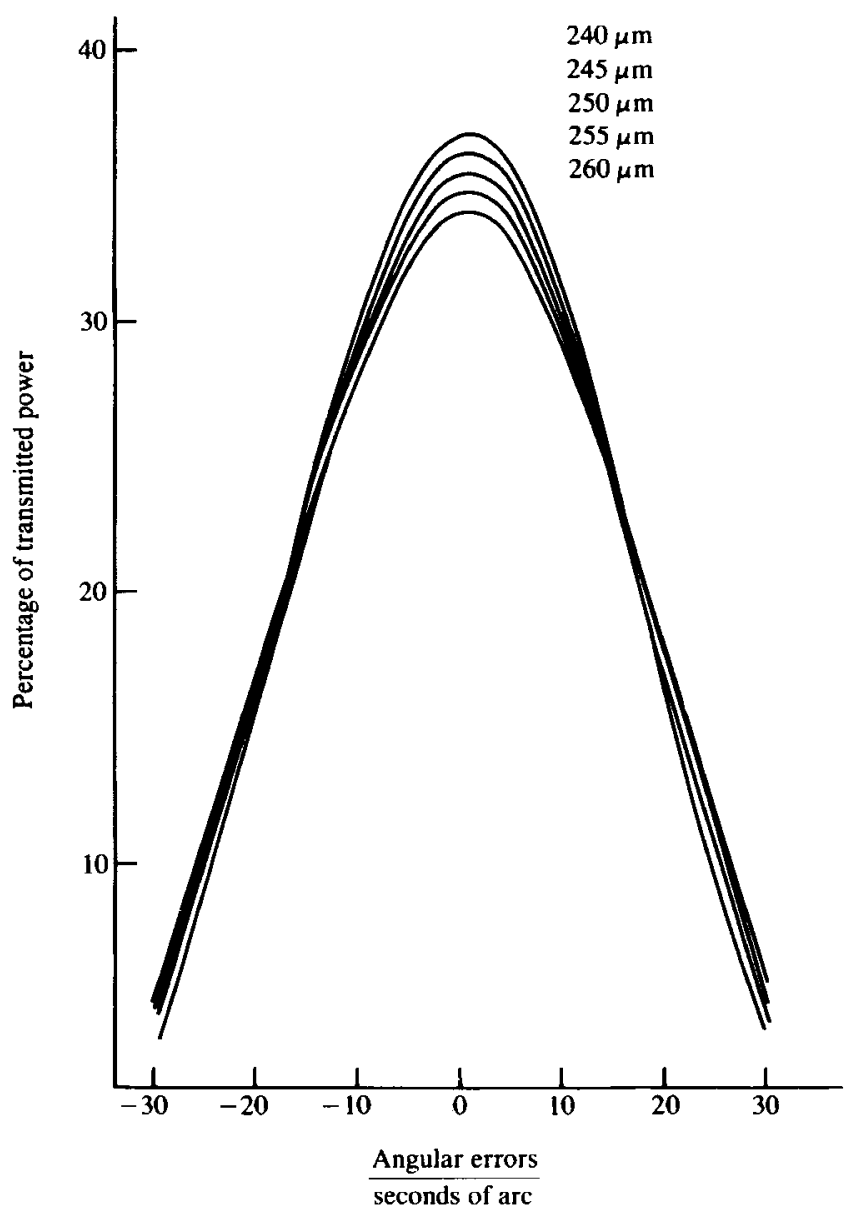

Fig. 7 Characteristic curve giving measurement sensitivity with angular errors $(10 \mu \mathrm{m}$ slit $)$

large values of misalignment and greater values of linear displacement, $z$. The latter point confirms the greater sensitivity of the device in the region of smaller translational errors as indicated by Fig. 3. Finer positional and orientational errors can be detected using the characteristic curves of Fig. 7.

\section{DISCUSSION AND CONCLUSIONS}

When any good quality reflective surface is mounted on a flat part of the robot gripper its position and pose with respect to the location of the sensing system can be determined to well below the normal range of the robot manipulative errors of $25-250 \mu \mathrm{m}$. For a particular orientation and position of the assembly fixtures with respect to the location of the sensing system, the errors in the position of the tool centre point of the gripper and the orientation of the end-effector with respect to the assembly site is ascertained. Inverse kinematics can then be used to correct the manipulator errors in order to ensure safe precision assembly to be performed. If these errors are of the order of the repeatability of the robotic manipulator, a micro-manipulator at the end of the wrist is required to exploit the precise measurement capability of the opto-electronic sensing device.

Owing to the differential detection principle employed, atmospheric factors such as vapour shimmer, humidity and smoke have a marginal influence on the detection accuracy of the device. Therefore, the device can operate under normal shop-floor environmental conditions without the need for regular maintenance or provisions of a clean room operation. The device is insensitive to mechanical vibrations and chatter of a target surface with peak to valley double amplitudes less than $5 \mu \mathrm{m}$. Performance of the instrument has not been practically verified yet. The normal target range is in the order of $10 \mathrm{~mm}$.

\section{REFERENCES}

1 Owen, A. E. Flexible assembly systems, 1984 (Plenum Press, London).

2 Heginbotham, W. B. Programmable assembly, 1986 (IFS Publications, Bedford)

3 Rosen C. A. and Nitzan, D. Use of sensors in programmable automation. Trans. IEEE., Comp. Soc. Magazine, December 1977, 12-23.

4 Chin, T. and Harlow, A. Automated visual inspection: a survey. Trans. IEEE. on Pattern Anal. and Mach. Intell., November 1982, 557-573.

5 Benhadj, R., Rahnejat, H. and Safa, M. M. A. A high resolution pneumatic proximity to tactile sensing device. Int. J. $A M T$, August 1987, 2(3), 59-72.

6 Foroughi, F., Rahnejat, H. and Bera, H. Tactile sensors for robot handling. Proc. Instn Mech. Engrs., Part B, 1987, 201 (B1), 51-58.

7 Ishii, M., Sakani, S., Kakikura, M. and Mikami, Y. A new 3D sensor for teaching robots paths and environment. Proc. Fourth International Conference on Robot Vision and Sensory Controls, December 1984 (IFS Conference, Bedford).

8 Mehdian, M. and Rahnejat, H. A tactile sensor with automatic learning capability for industrial parts inspection. Int. J. $A M T$, November 1987, 2(4), 11-26.

9 Masuda, R., Hasegawa K. and Osako, K. Slip sensor of industrial robot and its application. Elec. Engng in Japan, 1976, 96(5).

10 Light, B. Optical position sensing using silicon photodetectors. Lasers and Applications, 1986, V(4).

11 Kuntz, D. Specifying laser diode optics. Laser Focus, 1984, 20(3).

12 Fainman, Y., Lenz, E. and Shamir, J. Optical profilometer: a new method for high sensitivity and wide dynamic range. Applied Optics, September 1983, 21(17).

13 Kogelnik, H. Imaging of optical modes-resonators with internal lenses. Bell System Tech. Journal, March 1965.

14 Hull, D. M. and Stewart, A. Laser beam profiles--principles and definition. Laser and Applications, 1985, IV(10). 